\title{
Can a Health Checkup Record Predict the Onset of Reflux Esophagitis?
}

\author{
Kyoungwon Jung \\ Department of Internal Medicine, Kosin University College of Medicine, Busan, Korea
}

\begin{abstract}
Article: Predictive factors for future onset of reflux esophagitis: a longitudinal case-control study using health checkup records Toki $Y$, Yamauchi R, Kayashima E, et al (J Neurogastroenterol Motil 2022;28:86-94)
\end{abstract}

Gastroesophageal reflux disease (GERD) presenting as heartburn and acid regurgitation is one of the most common chronic gastrointestinal diseases, with a global prevalence of approximately 10-20\%. ${ }^{1,2}$ The pathophysiology of GERD has been continuously studied, and while not fully understood, it is certain that multiple factors contribute to its development. Taking into account all accepted concepts of pathophysiology, GERD is considered as a pathologic reflux that exceeds protective mechanisms, such as the esophagogastric junction barrier and esophageal acid clearance, both of which contribute to maintaining a physiologically balanced state. ${ }^{3}$ Dysfunction of such mechanisms eventually results in mucosal damage and reflux symptoms. Acid inhibitors, such as proton pump inhibitors, are the most vital component in GERD management. However, lifestyle modifications are considered to provide benefits similar to drug therapy. ${ }^{4}$ These include head elevation during bedtime, earlier dinnertimes, weight loss, smoking cessation, and avoidance of supine position at least 3 hours after meals. ${ }^{4}$

Lifestyle improvements are a vital part of GERD management, as several studies have associated the development of GERD with various demographic/environmental factors. ${ }^{2}$ Among these, obesity is most commonly reported and is considered a major risk factor for GERD. In a study reported by El-Serag et al, ${ }^{5}$ it was confirmed that as individuals progressed toward being overweight and obese, the occurrence of GERD symptoms and erosive esophagitis (EE) increased 2.5-fold. In addition, a meta-analysis also proved the association between obesity and GERD. ${ }^{6}$ In particular, it was confirmed that the risk of GERD symptoms increased with increasing body weight, with the risk being 1.43-fold for a body mass index (BMI) of $25-30 \mathrm{~kg} / \mathrm{m}^{2}$ and 1.94 -fold for a BMI of $>30 \mathrm{~kg} / \mathrm{m}^{2}{ }^{6}$. In the meta-analysis analyzing the effect of abdominal obesity, it was confirmed that the odds ratio of EE increased to 1.87. ${ }^{7}$ Other factors, such as metabolic syndrome (MetS), may also be related to EE. A study conducted on 7078 patients who underwent screening endoscopy confirmed that risk of reflux esophagitis increased by 1.42-fold in those with MetS. This particular finding proved that MetS also plays an important role in the development of GERD. ${ }^{8}$ In addition, a cross-sectional study of 507 patients conducted by Loke et al $^{9}$ noted that fasting blood sugar, triglyceride, aspartate aminotransferase, and alanine aminotransferase (ALT) levels were significantly higher in patients with $\mathrm{EE}$. Other associated risk factors mentioned in epidemiological studies include male sex, old age, smoking, drinking alcohol, and diabetes. ${ }^{2}$

A study conducted by Toki et al $^{10}$ utilized health examination data to identify factors predicting the occurrence of reflux esophagi-

Received: December 1, 2021 Revised: None Accepted: December 8, 2021

(.) This is an Open Access article distributed under the terms of the Creative Commons Attribution Non-Commercial License (http://creativecommons. org/licenses/by-nc/4.0) which permits unrestricted non-commercial use, distribution, and reproduction in any medium, provided the original work is properly cited.

${ }^{*}$ Correspondence: Kyoungwon Jung, MD

Department of Internal Medicine, Kosin University College of Medicine, 262 Gamcheon-ro, Seo-gu, Busan 49267, Korea

Tel: +82-51-990-6103, Fax: +82-51-990-5055, E-mail: forjkw@gmail.com 
tis (meaning of EE). In this study, matching analysis was performed on 2066 patients with endoscopy-confirmed EE and 4132 healthy controls. At the time of diagnosis, it was observed that BMI, ALT level, smoking history, acid reflux symptoms, hiatal hernia, and absence of atrophic gastritis were independently associated with EE. In addition, significant differences in BMI, abdominal circumference, fasting blood sugar, triglyceride, aspartate aminotransferase and ALT levels, percentage of acid reflux symptoms, and atrophic gastritis findings were still identified between the 2 groups at 5 years before the base year on longitudinal comparisons. The authors believe that these persistent differences may have had an effect on the occurrence of $\mathrm{EE}$.

One advantage of the study is that it is able to overcome the limitations of cross-sectional studies. To date, most studies have compared the differences between patients with and without GERD through baseline characteristics. ${ }^{5,89}$ In addition, several studies have validated this correlation by reducing the effects of risk factors, such as weight loss and smoking cessation, while improving symptoms and EE. ${ }^{11-13}$ However, direct determination of the effect of risk factors on the occurrence of GERD over time proved difficult because a majority of the studies were conducted over a short period of time or were only analyzed at the time of diagnosis or study. This longitudinal study was able to confirm that BMI, abdominal circumference, and blood test values, which are associated with obesity, MetS, fatty liver, and diabetes, were observed to increase over time and were found to be significantly worse in the EE patient group compared with the control group. ${ }^{10}$

However, this study faced certain limitations. One is that the timing of $\mathrm{EE}$ diagnosis in relation to the study period could not be verified. Another is the possibility of selective bias in reflecting the entire population since the study mainly utilized health checkup data of company employees. In addition, data on drug history were not accurately confirmed; thus, a risk group for EE that did not occur due to proton pump inhibitor treatment may have been included in the control group. Lastly, since the presence of EE defined the division between the patient and control groups, cases of non-erosive reflux disease (NERD) were not considered; hence, all cases of GERD were not included.

Nevertheless, this study was able to identify risk factors in patients with a potential risk of $\mathrm{EE}$ through utilization of health checkup data. In addition, changes with respect to time were analyzed. If long-term regional cohort studies are able to combine several validated questionnaires on GERD and health checkup data including the aforementioned risk factors, the influence of lifestyle on the occurrence of GERD, including NERD, may be quantified. Lastly, more studies are needed to determine whether these factors play a pathogenic role in the development of GERD.

\section{Financial support: None.}

Conflicts of interest: None.

\section{References}

1. Eusebi LH, Ratnakumaran R, Yuan Y, Solaymani-Dodaran M, Bazzoli F, Ford AC. Global prevalence of, and risk factors for, gastro-oesophageal reflux symptoms: a meta-analysis. Gut 2018;67:430-440.

2. Richter JE, Rubenstein JH. Presentation and epidemiology of gastroesophageal reflux disease. Gastroenterology 2018;154:267-276.

3. Herregods TV, Bredenoord AJ, Smout AJ. Pathophysiology of gastroesophageal reflux disease: new understanding in a new era. Neurogastroenterol Motil 2015;27:1202-1213.

4. Gyawali CP, Fass R. Management of gastroesophageal reflux disease. Gastroenterology 2018;154:302-318.

5. El-Serag HB, Graham DY, Satia JA, Rabeneck L. Obesity is an independent risk factor for GERD symptoms and erosive esophagitis. Am J Gastroenterol 2005;100:1243-1250.

6. Hampel H, Abraham NS, El-Serag HB. Meta-analysis: obesity and the risk for gastroesophageal reflux disease and its complications. Ann Intern Med 2005;143:199-211.

7. Singh S, Sharma AN, Murad MH, et al. Central adiposity is associated with increased risk of esophageal inflammation, metaplasia, and adenocarcinoma: a systematic review and meta-analysis. Clin Gastroenterol Hepatol 2013;11:1399-1412, e7.

8. Chung SJ, Kim D, Park MJ, et al. Metabolic syndrome and visceral obesity as risk factors for reflux oesophagitis: a cross-sectional case-control study of 7078 Koreans undergoing health check-ups. Gut 2008;57:13601365.

9. Loke SS, Yang KD, Chen KD, Chen JF. Erosive esophagitis associated with metabolic syndrome, impaired liver function, and dyslipidemia. World J Gastroenterol 2013;19:5883-5888.

10. Toki Y, Yamauchi R, Kayashima E, et al. Predictive factors for future onset of reflux esophagitis: a longitudinal case-control study using health checkup records. J Neurogastroenterol Motil 2022;28:86-94.

11. Ness-Jensen E, Hveem K, El-Serag H, Lagergren J. Lifestyle Intervention in Gastroesophageal Reflux Disease. Clin Gastroenterol Hepatol 2016;14:175-182, e1-e3.

12. Park SK, Lee T, Yang HJ, et al. Weight loss and waist reduction is associated with improvement in gastroesophageal disease reflux symptoms: a longitudinal study of 15295 subjects undergoing health checkups. Neurogastroenterol Motil 2017;29:e13009.

13. Kohata Y, Fujiwara Y, Watanabe T, et al. Long-term benefits of smoking cessation on gastroesophageal reflux disease and health-related quality of fife. PLoS One 2016;11:e0147860. 